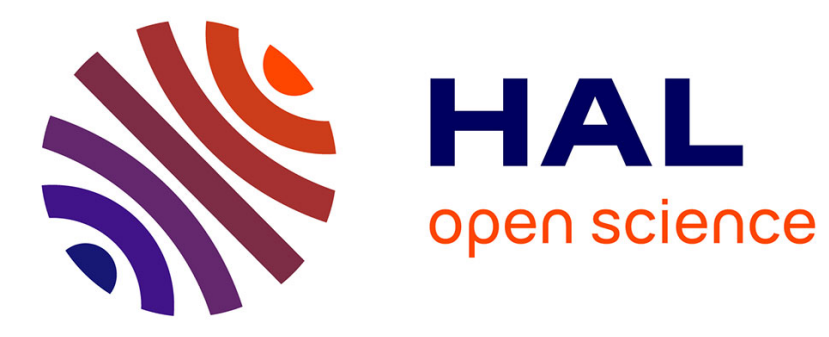

\title{
Preliminary orbital elements of six visual binary stars
}

\author{
M. Scardia, J.-L. Prieur, L. Pansecchi, R. W. Argyle
}

\section{To cite this version:}

M. Scardia, J.-L. Prieur, L. Pansecchi, R. W. Argyle. Preliminary orbital elements of six visual binary stars. Astronomical Notes / Astronomische Nachrichten, 2008, 329, pp.379. 10.1002/ASNA.200710966 . hal-00288770

\section{HAL Id: hal-00288770 \\ https://hal.science/hal-00288770}

Submitted on 20 Nov 2019

HAL is a multi-disciplinary open access archive for the deposit and dissemination of scientific research documents, whether they are published or not. The documents may come from teaching and research institutions in France or abroad, or from public or private research centers.
L'archive ouverte pluridisciplinaire HAL, est destinée au dépôt et à la diffusion de documents scientifiques de niveau recherche, publiés ou non, émanant des établissements d'enseignement et de recherche français ou étrangers, des laboratoires publics ou privés. 


\title{
Preliminary orbital elements of six visual binary stars.
}

\author{
M. Scardia ${ }^{1}$, J.-L. Prieur ${ }^{2}$, L. Pansecchi ${ }^{1}$, and R.W. Argyle ${ }^{3}$ \\ 1 INAF - Osservatorio Astronomico di Brera, Via E. Bianchi 46, 23807 Merate, Italy \\ 2 UMR 5572 d'Astrophysique, Observatoire Midi-Pyrénées - Université Paul Sabatier - CNRS, 14 Avenue \\ Edouard Belin, 31400 Toulouse, France \\ 3 Institute of Astronomy, Madingley Road, Cambridge, CB3 0HA, U.K.
}

Received Oct. 4, 2007; accepted

Key words Stars: binaries: visual — binaries: orbits — stars: masses — individual (ADS 1345, ADS 3610, ADS 4371, ADS 4376, ADS 7730, ADS 11956)

Preliminary new orbital elements were computed for the visual binary stars A 1 - ADS 1345, A 2629 ADS 3610, BU 560 - ADS 4371, STF 3115 - ADS 4376, STF 1426 AB - ADS 7730 and STF 2437 - ADS 11956. Using Straizys and Kuriliene's data, we derived new formulae for computing dynamical parallaxes for luminosity classes IV and V. The values found for those systems are in agreement with the Hipparcos parallaxes and the corresponding systemic masses are consistent with the spectral types.

\section{Introduction}

This paper deals with the determination of the orbital elements of six visual binary stars: A 1 - ADS 1345, A 2629 - ADS 3610, BU 560 - ADS 4371, STF 3115 ADS 4376, STF 1426 AB - ADS 7730 and STF 2437 ADS 11956. With the exception of ADS 3610, for which the declination $\left(\delta=-11^{\circ}\right)$ is too low, those objects belong to our sample of visual binaries that are regularly observed with the Pupil Interferometry Speckle camera and COronograph (PISCO) at the INAF - Osservatorio Astronomico di Brera (OAB) in Merate. PISCO is a speckle camera developed at Observatoire MidiPyrénées (Prieur at al. 1998) that was first used at Pic du Midi from 1993 to 1998. Since 2004 it has been mounted and operated at the Cassegrain focus of the INAF-OAB, 102-cm Zeiss telescope in Merate. Details about the data reduction procedure and the PISCO measurements used for this work can be found in Scardia et al. (2005, 2007a). The purpose of our long-term program is to provide good-quality measurements of close visual binaries in order to improve the quality of the orbits and derive estimates of the stellar masses.

In this paper we propose preliminary orbits for six systems. In Sect. 2 we describe the method used for computing the elements and derive mass estimates. In Sects. 3 to 8 we give more details and discuss the cases of each system separately. Finally, in Sect. 9 we compare our orbits of ADS 1345, 4376 and 7730 with those recently published by Novakovic (2006).

\section{Description of our method}

\subsection{Determination of the orbits}

For the calculation of the orbital elements we employed the same procedure as in the previous work of Scardia et al (2000). We first used the analytical method of Kowalsky (1873) to obtain preliminary values and then, when possible, improved them with the least-squares method of Hellerich (1925). The measurements were assigned a weight of $w$ defined as:

$$
w=\frac{c D_{\text {inch }}}{10 \sqrt{n}}
$$

where $D_{\text {inch }}$ is the diameter of the instrument, measured in inches, and $n$ is the number of nights used to derive this measurement. The coefficient $c$ is set to 1.0 for speckle observations and visual measurements made with refractor instruments, to 0.5 for the visual measurements made with reflector telescopes, and to 2.0 for photographic observations made with long-focal instruments. In the absence of any information about the measurements, we simply used a weight of $w=0.5$. This is the standard procedure that we have been using for more than thirty years for the determination of orbits, since it has proved to bring a good balance between the various sources of measurements.

The orbital elements that we have obtained for the six systems are presented in Table 1. Note that Hellerich's method only converged for ADS 3610, which explains why the errors could not be estimated for the other objects. In this table, $\Omega$ is the position angle of the ascending node, measured in the plane of the sky from north through east and $\omega$ is the longitude of the 
periastron in the plane of the true orbit, measured from the ascending node to the periastron, in the direction of motion of the companion, $i$ is the inclination of the orbit relative to the plane of the sky, $e$ the eccentricity, $T$ the epoch of periastron passage, $P$ the period, $n$ the mean angular motion, and $a$ is the semi-major axis. The four parameters A, B, F, and G are the Thiele-Innes constants.

The corresponding ephemerides for the years from 2007.0 to 2016.0 are given in Table 2 with, for each binary, the separation $\rho$ in arcsec and the position angle $\theta$ in degrees.

The corresponding $O-C$ (Observed minus Computed) residuals are displayed in Tables 4 to 9 , with the date in years (col. 1), the observed separation $\rho$ in $\operatorname{arcsec}(\operatorname{col} .2), \Delta \rho(O-C)$ in $\operatorname{arcsec}(\operatorname{col} .3)$, the observed position angle $\theta$ (reduced to 2000) in degrees (col. 4), $\Delta \theta(O-C)$ in degrees (col. 5), the number of independent observations (col. 6) and the name of the observer (col. 7) as it appears in the Washington Double Star Catalogue (Mason et al. 2007). All the tables, with the exception of Table 5, are restricted for reasons of space to the observations made since 1991.0.

The apparent orbits are plotted in solid lines in Fig. 2 and the measurements used for the calculation of the orbital elements are represented with small crosses. For ADS 1345, 4376 and 7730, the three orbits proposed by Novakovic (2006) are displayed as dotted lines, for comparison. The location of the primary component is indicated with a big cross $(+)$ and the line of apsides is drawn as a straight segment going through this point. The orientation of the plots conforms with the convention accepted by the observers of visual binary stars. The sense of motion of the companion is indicated with an arrow.

\subsection{Mass determination}

Most of the errors in the masses derived from orbits of visual binaries come from the poor determination of the parallax, and whilst Hipparcos measurements are very useful, they often have a large uncertainty. Indeed, as the typical errors on Hipparcos measurements are $\sigma_{\text {Hip }} \approx 1$ mas, the relative errors are larger than $10 \%$ for the systems located at distances larger than 100 pc. The application of Kepler's third law to a binary system leads to

$$
\mathfrak{M}_{\text {syst }}=(a / p)^{3} / P^{2}
$$

where $\mathfrak{M}_{\text {syst }}$ is the "systemic mass" in $\mathfrak{M}_{\odot}\left(\mathfrak{M}_{\text {syst }}=\right.$ $\mathfrak{M}_{1}+\mathfrak{M}_{2}$ is the sum of the masses of the two components), a the angular semi-major axis in arcsec, $p$ the parallax in arcsec, and $P$ the period in years. The logarithmic derivative of Eq. 2 gives:

$$
\frac{d \mathfrak{M}_{\text {syst }}}{\mathfrak{M}_{\text {syst }}}=3 \frac{d a}{a}-2 \frac{d P}{P}-3 \frac{d p}{p}
$$

Hence to obtain a relative error smaller than $10 \%$ for the masses, the error on the parallax should be smaller than $3.3 \%$. Unfortunately the Hipparcos satellite could only measure 90 visual binaries with this precision. Future astrometric satellites are needed to improve the accuracy of the masses derived from orbits of visual binaries.

We display in Table 3 some data about the six systems studied here, with the spectral types of the global systems found in the CDS SIMBAD data base (http://simbad.u-strasbg.fr) and in Duflot et al. (1995) in line 2, the apparent $V$ magnitudes of the primary $\left(m_{V 1}\right)$ and secondary $\left(m_{V 2}\right)$ stars in lines 3 and 4 , respectively, and the Hipparcos parallaxes $p_{\text {Hip }}$ (ESA, 1997) with their errors in line 5.

For each system, we computed the absolute magnitude $M_{V 1, \text { Hip }}$ (in line 6 ) from $m_{V 1}$ and $p_{\text {Hip }}$. We assumed that the interstellar absorption was negligible, which is justified by the small distances of the objects. We could then estimate the luminosity classes of the primary stars (Class(Hip.) in line 7) by comparing $M_{V 1 \text {,Hip }}$ with the expected theoretical values (from Schmidt-Kaler, 1982), assuming that the global spectral type could be attributed to the primary star. The Hipparcos parallax of ADS 7730 has a large uncertainty and the deduced luminosity class of its primary is badly determined. But for the other stars, the Hipparcos measurements are more precise and imply they belong to classes IV or V.

For each system, we computed $p_{\text {dyn }}$, the dynamical parallax, using Baize-Romani (1945)'s method (see also Couteau, 1978), with some modifications. Indeed this method normally applies only to main sequence (i.e. class V) stars and assumes that the two components follow the mass-luminosity relation:

$$
\log _{10} \mathfrak{M}=-k\left(M_{\mathrm{bol}}-M_{0}\right)
$$

where $\mathfrak{M}$ is the mass of the star (in $\mathfrak{M}_{\odot}$ ), $M_{\text {bol }}$ is its absolute bolometric magnitude, and $k$ and $M_{0}$ are two constants.

With the data available at that time, Baize (1947) found $k=0.1117$ and $M_{0}=4.77$ (i.e. the bolometric magnitude of the Sun) which are the values used in the Baize-Romani (1945)'s formulae. To revise those values with more recent data, we fitted the relation (3) to Straizys \& Kuriliene (1981)'s data for the classes $\mathrm{V}$ and IV (in the range B0-M5), and obtained $\left(k=0.1045, M_{0}=4.60\right)$ and $\left(k=0.1157, M_{0}=3.75\right)$, respectively. The corresponding residuals are 0.45 and 0.19 for the bolometric magnitudes. The fitted lines are displayed in Fig. 1. Note that this is only a simple approximation, especially for class IV, whose contours are rather fuzzy in the HR diagram.

A more elaborated model of the mass-luminosity relation for stars of class $\mathrm{V}$ was proposed by Angelov (1993), with a 7th order polynomial instead of the first 
Table 1 Orbital elements of the six new orbits

\begin{tabular}{|c|c|c|c|c|c|c|c|c|c|c|c|c|}
\hline $\begin{array}{l}\text { Name } \\
\text { (ADS) }\end{array}$ & $\begin{array}{c}\Omega \\
(2000)\end{array}$ & $\begin{array}{c}\omega \\
\left(^{\circ}\right)\end{array}$ & $\begin{array}{c}i \\
\left({ }^{\circ}\right)\end{array}$ & $e$ & $\begin{array}{c}T \\
(\mathrm{yr})\end{array}$ & $\begin{array}{c}P \\
(\mathrm{yr})\end{array}$ & $\begin{array}{c}n \\
(\% / \mathrm{yr})\end{array}$ & $\begin{array}{c}a \\
\left({ }^{\prime \prime}\right)\end{array}$ & $\begin{array}{c}\mathrm{A} \\
\left({ }^{\prime \prime}\right)\end{array}$ & $\begin{array}{c}\mathrm{B} \\
\left({ }^{\prime \prime}\right)\end{array}$ & $\begin{array}{c}\mathrm{F} \\
\left({ }^{\prime \prime}\right)\end{array}$ & $\begin{array}{c}\mathrm{G} \\
\left({ }^{\prime \prime}\right)\end{array}$ \\
\hline 1345 & 163.1 & 303.0 & 44.1 & 0.719 & 1885.316 & 676.7 & 0.53199 & 0.989 & -0.34223 & 0.72651 & -0.90607 & -0.12899 \\
\hline 3610 & $\begin{array}{l}108.2 \\
\pm 3.4\end{array}$ & $\begin{array}{c}104.3 \\
\pm 3.1\end{array}$ & $\begin{array}{l}115.2 \\
\pm 3.8\end{array}$ & $\begin{array}{c}0.844 \\
\pm 0.026\end{array}$ & $\begin{array}{c}1958.143 \\
\pm 0.15\end{array}$ & $\begin{array}{l}154.7 \\
\pm 13.5\end{array}$ & $\begin{array}{c}2.32708 \\
\pm 0.20\end{array}$ & $\begin{array}{c}0.187 \\
\pm 0.014\end{array}$ & 0.08756 & -0.01985 & 0.03767 & -0.17785 \\
\hline 4371 & 139.5 & 242.5 & 129.7 & 0.644 & 1860.665 & 811.2 & 0.44379 & 1.520 & -0.02562 & -1.11070 & -1.31639 & 0.53471 \\
\hline 4376 & 89.1 & 201.6 & 141.0 & 0.633 & 2106.0 & 1370.0 & 0.26277 & 1.585 & -0.47654 & -1.46639 & -1.13597 & 0.60139 \\
\hline 7730 & 114.0 & 22.8 & 47.2 & 0.189 & 1674.230 & 661.8 & 0.54395 & 0.800 & -0.49239 & 0.58806 & -0.33167 & -0.48702 \\
\hline 11956 & 114.9 & 212.1 & 131.0 & 0.478 & 2132.947 & 804.6 & 0.44743 & 0.910 & 0.03681 & -0.83280 & -0.66233 & 0.22569 \\
\hline
\end{tabular}

Table 2 Ephemerides computed with the six new orbits.

\begin{tabular}{|c|c|c|c|c|c|c|c|c|c|c|c|c|}
\hline \multirow[b]{2}{*}{ Epoch } & \multicolumn{2}{|c|}{ ADS 1345} & \multicolumn{2}{|c|}{ ADS 3610} & \multicolumn{2}{|c|}{ ADS 4371} & \multicolumn{2}{|c|}{ ADS 4376} & \multicolumn{2}{|c|}{ ADS 7730} & \multicolumn{2}{|c|}{ ADS 11956} \\
\hline & $\begin{array}{c}\rho \\
\left({ }^{\prime \prime}\right)\end{array}$ & $\begin{array}{c}\theta \\
\left(^{\circ}\right)\end{array}$ & $\begin{array}{c}\rho \\
\left({ }^{\prime \prime}\right)\end{array}$ & $\begin{array}{c}\theta \\
\left({ }^{\circ}\right)\end{array}$ & $\begin{array}{c}\rho \\
\left({ }^{\prime \prime}\right)\end{array}$ & $\begin{array}{c}\theta \\
\left(^{\circ}\right)\end{array}$ & $\begin{array}{c}\rho \\
\left({ }^{\prime \prime}\right)\end{array}$ & $\begin{array}{c}\theta \\
\left(^{\circ}\right)\end{array}$ & $\begin{array}{c}\rho \\
\left({ }^{\prime \prime}\right)\end{array}$ & $\begin{array}{c}\theta \\
\left({ }^{\circ}\right)\end{array}$ & $\begin{array}{c}\rho \\
\left({ }^{\prime \prime}\right)\end{array}$ & $\begin{array}{c}\theta \\
\left(^{\circ}\right)\end{array}$ \\
\hline 2007.0 & 0.839 & 250.7 & 0.134 & 190.8 & 1.645 & 126.0 & 0.814 & 340.2 & 0.910 & 310.5 & 0.575 & 10.8 \\
\hline 2008.0 & 0.843 & 251.1 & 0.135 & 189.8 & 1.650 & 125.8 & 0.811 & 339.6 & 0.908 & 310.7 & 0.573 & 10.2 \\
\hline 2009.0 & 0.847 & 251.5 & 0.137 & 188.8 & 1.655 & 125.6 & 0.807 & 339.0 & 0.907 & 311.0 & 0.572 & 9.5 \\
\hline 2010.0 & 0.851 & 251.8 & 0.138 & 187.8 & 1.659 & 125.4 & 0.804 & 338.4 & 0.905 & 311.3 & 0.571 & 8.9 \\
\hline 2011.0 & 0.854 & 252.2 & 0.139 & 186.9 & 1.664 & 125.3 & 0.800 & 337.8 & 0.904 & 311.6 & 0.569 & 8.2 \\
\hline 2012.0 & 0.858 & 252.5 & 0.140 & 185.9 & 1.668 & 125.1 & 0.797 & 337.2 & 0.903 & 311.9 & 0.568 & 7.5 \\
\hline 2013.0 & 0.862 & 252.9 & 0.141 & 185.0 & 1.673 & 124.9 & 0.793 & 336.5 & 0.901 & 312.2 & 0.567 & 6.9 \\
\hline 2014.0 & 0.865 & 253.2 & 0.143 & 184.1 & 1.677 & 124.7 & 0.790 & 335.9 & 0.900 & 312.5 & 0.565 & 6.2 \\
\hline 2015.0 & 0.869 & 253.6 & 0.144 & 183.1 & 1.682 & 124.5 & 0.786 & 335.3 & 0.898 & 312.7 & 0.564 & 5.5 \\
\hline 20160 & 0.873 & 253.9 & 0.145 & 182.3 & 1.686 & 124.4 & 0.783 & 334.6 & 0.897 & 313.0 & 0.563 & 4.9 \\
\hline
\end{tabular}

Table 3 Characteristics of the studied systems. The subscripts 1 and 2 refer to the primary and secondary components, respectively.

\begin{tabular}{llcccccc}
\hline$(1)$ & ADS & 1345 & 3610 & 4371 & 4376 & 7730 & 11956 \\
\hline$(2)$ & Spectral type & F2 & A0 & F8 & A2-4V & F5-7 & G5 \\
$(3)$ & $m_{V 1}(\mathrm{mag})$ & 8.75 & 7.73 & 7.77 & 6.55 & 7.99 & 8.41 \\
$(4)$ & $m_{V 2}(\mathrm{mag})$ & 8.90 & 8.63 & 8.24 & 7.51 & 8.30 & 8.83 \\
$(5)$ & $p_{\text {Hip }}(\mathrm{mas})$ & 7.74 & 4.30 & 12.21 & 7.91 & 6.98 & 12.15 \\
& & \pm 3.57 & \pm 0.87 & \pm 1.88 & \pm 0.92 & \pm 5.95 & \pm 2.46 \\
$(6)$ & $M_{V 1, \text { Hip }}(\mathrm{mag})$ & 3.2 & 0.9 & 3.2 & 1.0 & 2.2 & 3.8 \\
& & \pm 1.1 & \pm 0.5 & \pm 0.3 & \pm 0.3 & \pm 2.8 & \pm 0.5 \\
$(7)$ & Class $($ Hip. $)$ & $\mathrm{IV}-\mathrm{V}$ & $\mathrm{V}$ & $\mathrm{IV}$ & $\mathrm{IV}$ & $\mathrm{III}-\mathrm{V}$ & $\mathrm{IV}$ \\
$(8)$ & $q\left(=\mathfrak{M}_{2} / \mathfrak{M}_{1}\right)$ & 0.96 & 0.81 & 0.88 & 0.77 & 0.92 & 0.89 \\
$(9)$ & $p_{\text {dyn }}(\mathrm{mas})$ & 9.9 & 3.8 & 13.8 & 8.4 & 7.5 & 7.8 \\
$(10)$ & $(1+q) \mathfrak{M}_{\text {theo }}\left(\mathfrak{M}_{\odot}\right)$ & 3.7 & 4.1 & 2.4 & 3.7 & 2.6 & 2.3 \\
$(11)$ & $\mathfrak{M}_{\text {syst }}\left(\mathfrak{M}_{\odot}\right)$ & 4.6 & 3.4 & 2.9 & 4.3 & 3.4 & 0.6 \\
$(12)$ & $a(\mathrm{AU})$ & 128 & 43 & 124 & 200 & 115 & 75 \\
\hline
\end{tabular}

order polynomial of Eq. 3. This allows a reduction of the residuals of the fit, especially for very small mass stars, but it makes the derivation of dynamical parallaxes much more complex. As shown in Fig. 1, our simple model is satisfactory for the classes $\mathrm{V}$ and $\mathrm{IV}$ in the range B0-M5. We have thus used it as it allows a straightforward determination of $p_{\text {dyn }}$ as we will see below.

Using the values of $k$ found for Eq. 3, we derived the ratio $q=\mathfrak{M}_{2} / \mathfrak{M}_{1}$ from the difference of magnitudes of the two components: $q=10^{-k \Delta m_{\mathrm{bol}}}$. For the systems studied here, we assumed that $\Delta m_{\text {bol }} \approx \Delta m_{V}$, and obtained the values of $q$ given in line 8 of Table 3 .
After some arithmetic, the combination of Eqs. 2, 3 and Pogson's relation leads to:

$$
\begin{aligned}
(3-5 k) \log _{10} p= & \log _{10}\left(a^{3} / P^{2}\right)-\log _{10}(1+q) \\
& +k\left(m_{\mathrm{V} 1}+5+C_{\mathrm{V} 1}-M_{0}\right)
\end{aligned}
$$

where $m_{V 1}$ and $C_{V 1}$ are the visual magnitude and the bolometric correction of the primary star. The parallax obtained in this way is called dynamical parallax, and will be noted in the following as $p_{\text {dyn }}$. Using the values 
of $k$ and $M_{0}$ found for classes $\mathrm{V}$ and IV we derive:

$$
\begin{aligned}
\log _{10} p_{\mathrm{dyn}}= & 0.017+1.211 \log _{10} \alpha-0.404 \log _{10}(1+q) \\
& +0.042\left(m_{\mathrm{V} 1}+C_{\mathrm{V} 1}\right) \quad(\text { for class } \mathrm{V}) \\
\log _{10} p_{\mathrm{dyn}}= & 0.060+1.239 \log _{10} \alpha-0.413 \log _{10}(1+q) \\
& +0.048\left(m_{\mathrm{V} 1}+C_{\mathrm{V} 1}\right) \quad(\text { for class IV })
\end{aligned}
$$

where $\alpha=a P^{-2 / 3}$.

The dynamical parallaxes derived with those formulae are reported in line 9 of Table 3. For ADS 1345, we computed the mean of the two values of $p_{\text {dyn }}$ corresponding to classes IV and V. For ADS 7730, we assumed a luminosity class equal to IV. The values found are in good agreement with the Hipparcos measurements. The largest discrepancy, for ADS 11956, corresponds to a difference of $1.8 \sigma_{\mathrm{Hip}}$, where $\sigma_{\mathrm{Hip}}$ is the error of the Hipparcos parallax. For the other objects, the differences are smaller than $\sigma_{\text {Hip }}$.

In Sects. 3 to 8, we will compare the systemic masses $\mathfrak{M}_{\text {syst }}$ (line 11) derived from our orbits using the Hipparcos parallaxes with $(1+q) \mathfrak{M}_{\text {theo }}$ (line 10), where $\mathfrak{M}_{\text {theo }}$ is the typical theoretical mass from Straizys \& Kuriliene (1981) of a star whose spectral type and luminosity class are given in lines 2 and 7 , respectively.

Finally we reported in line 12 of Table 3 the linear values of $a$ in AU derived from our orbits with the Hipparcos parallaxes.

\section{WDS 01424 -0645 - A 1 - ADS 1345}

This binary was discovered by R.G. Aitken in 1899 with the 36-inch refractor of the Lick Observatory, and recently observed by PISCO at OAB in 2006. The available observations cover an arc of about $90^{\circ}$, which seems sufficient to attempt a calculation of a new set of orbital elements. The old orbit by Erceg (1981) no longer represents the observations, so we have recalculated the orbital elements and obtained the values reported in Table 1. As shown in Fig. 2a, the orbital motion is still poorly covered by the observations and our orbit is to be considered as preliminary only.

The systemic mass derived from our orbit with the Hipparcos parallax is $4.6 \mathfrak{M}_{\odot}$, which is slightly in excess of that expected for a binary of spectral type F2 IV$\mathrm{V}$, i.e. $3.7 \mathfrak{M}_{\odot}$ (see Table 3 ). It should be noted that the Hipparcos parallax has a large uncertainty though (about 50\%).

\section{WDS 05010 -1112 - A 2629 - ADS 3610}

This binary was discovered by R.G. Aitken in 1913 with the 36-inch refractor of the Lick Observatory. It is a very close pair, which is difficult to observe visually ( $\left.\rho \approx 0^{\prime \prime} .1\right)$. This explains why one of us (M. Scardia)
Table $4 O-C$ residuals for the new orbit of A 1 - ADS 1345.

\begin{tabular}{rcrcrll}
\hline Epoch & $\begin{array}{c}\rho \\
\left({ }^{\prime \prime}\right)\end{array}$ & $\begin{array}{c}\Delta \rho_{(O-C)}\left({ }^{\prime \prime}\right) \\
\end{array}$ & $\begin{array}{c}\theta \\
\left({ }^{\circ}\right)\end{array}$ & $\begin{array}{r}\Delta \theta_{(O-C)}\left({ }^{\circ}\right) \\
\text { n }\end{array}$ & Observer \\
\hline 1991.250 & 0.783 & 0.004 & 243.9 & -0.5 & 1 & HIP \\
1991.550 & 0.780 & 0.000 & 244.2 & -0.4 & 1 & TYC \\
1991.713 & 0.787 & 0.006 & 244.2 & -0.4 & 1 & HRT \\
1995.772 & 0.750 & -0.046 & 244.4 & -2.0 & 1 & WSI \\
1995.918 & 0.789 & -0.008 & 245.4 & -1.0 & 1 & HRT \\
1996.040 & 0.720 & -0.077 & 252.0 & 5.5 & 2 & HEI \\
1996.901 & 0.811 & 0.010 & 246.9 & 0.1 & 1 & HOR \\
1997.039 & 0.780 & -0.021 & 245.7 & -1.2 & 1 & WSI \\
1999.773 & 0.810 & -0.002 & 246.1 & -1.9 & 1 & HOR \\
1999.781 & 0.860 & 0.048 & 244.6 & -3.4 & 1 & HOR \\
1999.784 & 0.850 & 0.038 & 251.7 & 3.7 & 1 & HOR \\
1999.795 & 0.860 & 0.048 & 245.5 & -2.5 & 1 & HOR \\
2001.868 & 0.780 & -0.040 & 249.9 & 1.1 & 1 & WSI \\
2001.876 & 0.840 & 0.020 & 248.6 & -0.2 & 1 & HOR \\
2001.876 & 0.843 & 0.023 & 247.3 & -1.5 & 1 & HOR \\
2003.831 & 0.790 & -0.037 & 250.3 & 0.8 & 3 & WSI \\
2003.960 & 0.796 & -0.032 & 248.5 & -1.1 & 1 & MSN \\
2006.091 & 0.848 & 0.012 & 249.5 & -0.9 & 1 & SCA \\
\hline
\end{tabular}

failed to resolve it in 1987 with the $38-\mathrm{cm}$ G.P.O. astrograph of La Silla (E.S.O. - Chile), utilized as a visual refractor (Scardia, 1991). The last positive observation was made ten years ago. Very skilled observers, such as Aitken, Voûte, van den Bos, Finsen and Worley, reported that the two components of the system had the same magnitude. This is in disagreement with Hipparcos observations, which report a difference of 0.9 mag. (however this measurement is doubtful since $\rho$ was estimated to be only $0^{\prime \prime} .122$ in 1991).

The orbital elements given in Table 1 were obtained with the assumption that all the published quadrants were correct. Three observations, namely B (1958.06), FIN (1963.13) and HIP (1991.25), appeared as aberrant and had to be rejected for this computation. Considering the difficulty of measuring this couple, the representation of the observations seems reasonable. The Hipparcos parallax leads to a systemic mass of $3.4 \mathfrak{M}_{\odot}$, with an uncertainty of $67 \%$, a little in default for a system of spectral type $\mathrm{A} 0 \mathrm{~V}\left(4.1 \mathfrak{M}_{\odot}\right.$ in line 10 of Table 3).

If future observations confirm that the two stars have the same magnitude, as reported by visual observers, it will be necessary to consider changing the quadrant of the observations made after 1956. This would lead to a longer period orbit, which seems to be suggested by the general trend of the observations. Therefore, our orbit (Fig. 2b) is to be considered as provisional. 

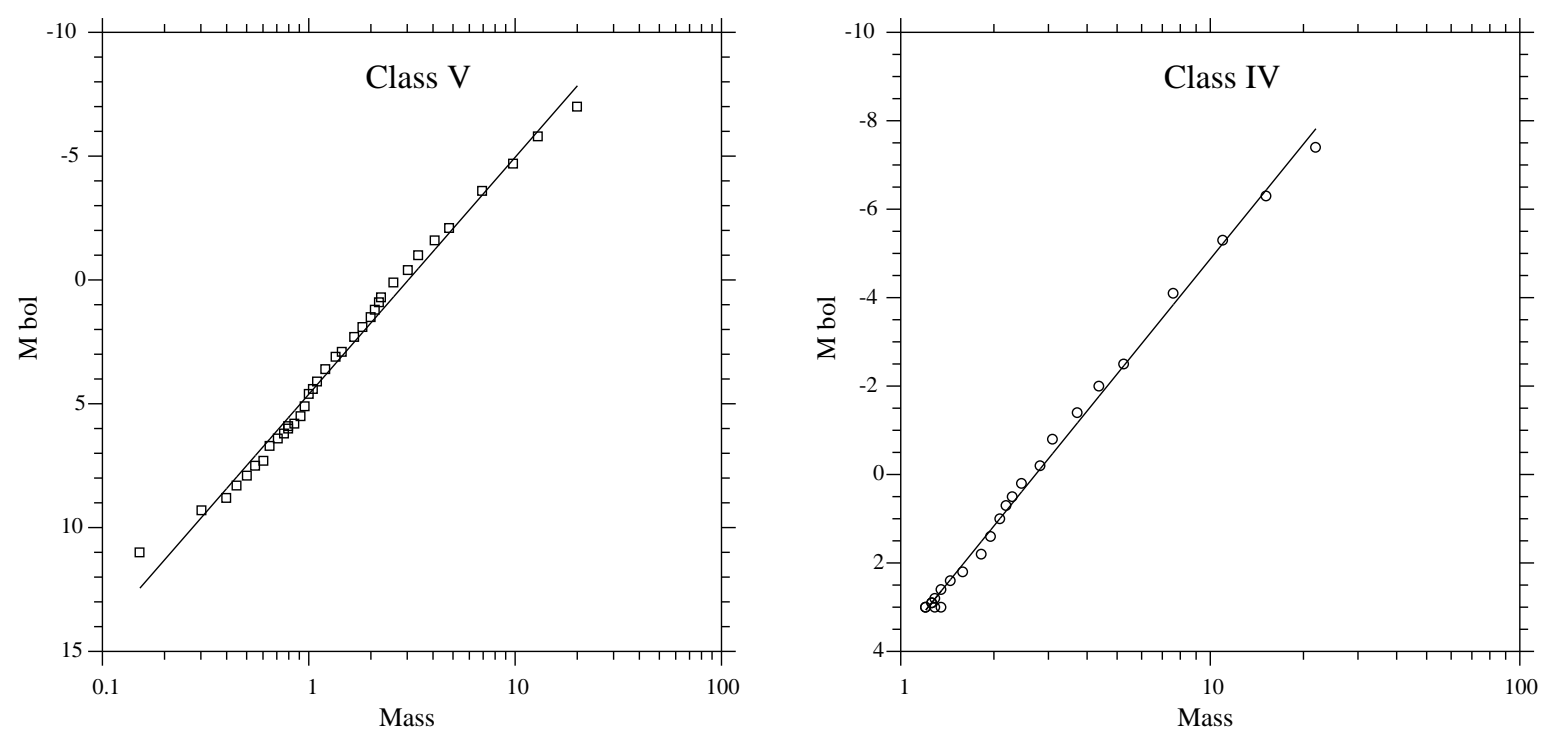

Fig. 1 Determination of mass-luminosity relation for luminosity classes V and IV with Straizys \& Kuriliene (1981)'s data.

Table $5 \quad O-C$ residuals for the new orbit of A 2629 ADS 3610.

\begin{tabular}{rcrrrll}
\hline Epoch & $\begin{array}{c}\rho \\
\left({ }^{\prime \prime}\right)\end{array}$ & $\begin{array}{c}\Delta \rho_{(\mathrm{O}-C)}\left({ }^{\prime \prime}\right) \\
\left(^{\circ}\right)\end{array}$ & $\begin{array}{r}\Delta \theta_{(O-C)}\left({ }^{\circ}\right) \\
\text { n }\end{array}$ & Observer \\
\hline 1913.920 & 0.220 & 0.040 & 148.1 & 0.7 & 2 & A \\
1921.810 & 0.190 & 0.014 & 141.9 & -0.8 & 3 & $\mathrm{~A}$ \\
1932.920 & 0.170 & 0.007 & 134.4 & -1.3 & 1 & $\mathrm{~A}$ \\
1944.830 & 0.120 & -0.011 & 127.0 & 1.4 & 1 & VBS \\
1957.780 & 0.150 & 0.137 & 27.2 & -0.4 & 2 & MLR \\
1960.130 & 0.116 & 0.072 & 291.7 & 3.3 & 2 & FIN \\
1978.796 & 0.110 & 0.011 & 227.1 & -3.2 & 2 & WOR \\
1989.933 & 0.104 & -0.009 & 211.9 & 0.0 & 1 & HRT \\
1990.916 & 0.106 & -0.008 & 211.6 & 1.2 & 1 & HRT \\
1990.924 & 0.106 & -0.008 & 210.9 & 0.5 & 1 & HRT \\
1993.092 & 0.108 & -0.009 & 207.6 & 0.3 & 1 & HRT \\
1995.921 & 0.111 & -0.009 & 203.0 & -0.6 & 1 & HRT \\
1996.863 & 0.116 & -0.005 & 201.2 & -1.2 & 1 & HRT \\
\hline
\end{tabular}

\section{WDS 05474 +2939 - BU 560 - ADS 4371}

This system was discovered by S.W. Burnham in November 1877 with the 18.5-inch refractor of the Dearborn Observatory in Chicago and has been well observed since then. Since its discovery, the companion has described an arc of more than $80^{\circ}$, which is sufficient for the computation of a preliminary orbit. The last two observations were made with the speckle camera PISCO at OAB. Our orbit is represented in Fig. 2c. The systemic mass derived with Hipparcos parallax would be $2.9 \mathfrak{M}_{\odot}$, which is very close to the value expected for a binary star of spectral type F8 IV, i.e., $2.4 \mathfrak{M}_{\odot}$ (see line 10 of Table 3 ).
Table $6 O-C$ residuals for the new orbit of BU 560 ADS 4371

\begin{tabular}{ccrrrll}
\hline Epoch & $\begin{array}{c}\rho \\
\left({ }^{\prime \prime}\right)\end{array}$ & $\begin{array}{r}\Delta \rho_{(O-C)}\left({ }^{\prime \prime}\right) \\
\left({ }^{\circ}\right)\end{array}$ & $\begin{array}{r}\Delta \theta_{(O-C)}\left({ }^{\circ}\right) \\
\text { n }\end{array}$ & Observer \\
\hline 1991.095 & 1.570 & 0.007 & 129.7 & 0.6 & 1 & WSI \\
1991.109 & 1.590 & 0.027 & 129.6 & 0.5 & 1 & WSI \\
1991.114 & 1.520 & -0.043 & 129.2 & 0.1 & 1 & WSI \\
1991.117 & 1.560 & -0.003 & 129.2 & 0.1 & 1 & WSI \\
1991.161 & 1.580 & 0.017 & 129.7 & 0.6 & 1 & WSI \\
1991.250 & 1.597 & 0.033 & 129.3 & 0.2 & 1 & HIP \\
1991.620 & 1.598 & 0.032 & 129.5 & 0.5 & 1 & TYC \\
1994.134 & 1.600 & 0.020 & 129.8 & 1.4 & 1 & WSI \\
1994.159 & 1.560 & -0.020 & 128.8 & 0.4 & 1 & WSI \\
1995.770 & 1.580 & -0.009 & 128.3 & 0.2 & 1 & WSI \\
1995.781 & 1.560 & -0.029 & 128.1 & -0.0 & 1 & WSI \\
1999.108 & 1.580 & -0.026 & 128.6 & 1.1 & 1 & WSI \\
2004.007 & 1.520 & -0.111 & 126.5 & -0.1 & 1 & LOS \\
2005.218 & 1.643 & 0.006 & 126.2 & -0.1 & 1 & SCA \\
2007.083 & 1.653 & 0.007 & 125.3 & -0.7 & 1 & SCA \\
\hline
\end{tabular}

\section{WDS 05491 +6248 - STF 3115 - ADS 4376}

This pair was discovered by F.G.W. Struve in 1831, after the publication of the "Catalogus Novus" (Struve, 1827), during a campaign of observations of the double stars already catalogued. It was then added, with 21 other new objects, to the list of the double stars that bear the name of Struve in the table "Supplementum II", published in the "Mensurae Micrometricae" (Struve, 1837). The primary star, HD 38284, is a fast rotator with $v \sin i=159 \mathrm{~km} . \mathrm{s}^{-1}$ (Royer et al. 2002). Duflot et al. (1995) reports a radial velocity of $-4 \mathrm{~km} . \mathrm{s}^{-1}$ and a spectral type of A2 V. It was observed 

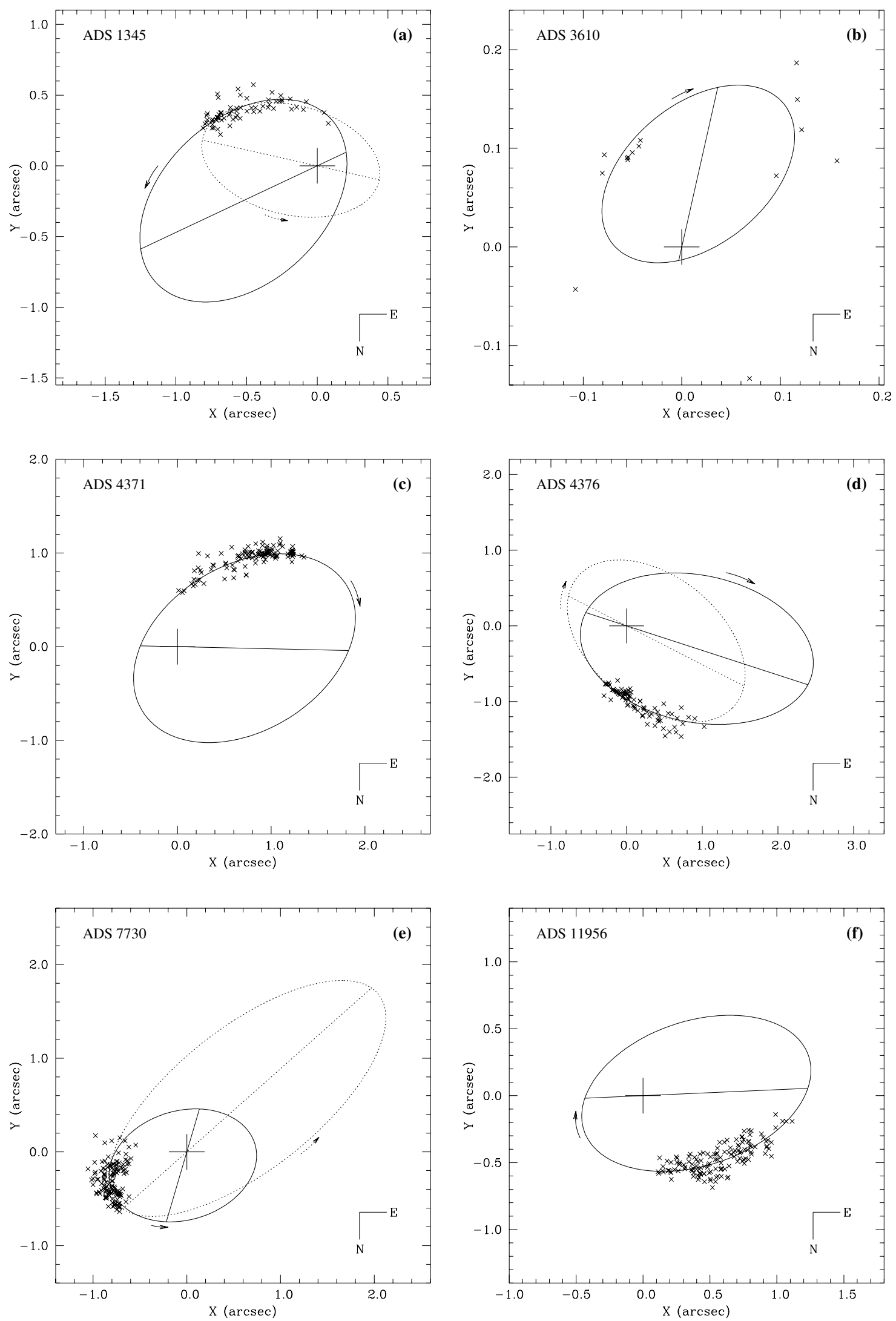

Fig. 2 New orbits (in solid lines) proposed for A 1 - ADS 1345 (a), A 2629 - ADS 3610 (b), BU 560 - ADS 4371 (c), STF 3115 - ADS 4376 (d), STF 1426 AB - ADS 7730 (e) and STF 2437 - ADS 11956 (f). For ADS 1345,4376 and 7730 , we also displayed in dotted lines the orbits computed by Novakovic (2006). 
Table $7 \quad O-C$ residuals for the new orbit of STF 3115 - ADS 4376

\begin{tabular}{rcrcrll}
\hline Epoch & $\begin{array}{c}\rho \\
\left({ }^{\prime \prime}\right)\end{array}$ & $\begin{array}{c}\Delta \rho_{(O-C)}\left({ }^{\prime \prime}\right) \\
\left({ }^{\circ}\right)\end{array}$ & $\begin{array}{c}\Delta \theta_{(O-C)}\left({ }^{\circ}\right) \\
\text { n }\end{array}$ & Observer \\
\hline 1991.250 & 0.861 & -0.013 & 348.4 & -0.6 & 1 & HIP \\
1991.790 & 0.869 & -0.003 & 347.4 & -1.3 & 1 & TYC \\
1991.894 & 0.858 & -0.014 & 348.2 & -0.5 & 1 & HRT \\
1992.170 & 1.000 & 0.130 & 348.0 & -0.5 & 3 & MLR \\
1994.790 & 0.730 & -0.130 & 350.7 & 3.5 & 3 & ALZ \\
2000.178 & 0.810 & -0.029 & 344.0 & -0.2 & 1 & WSI \\
2002.995 & 0.810 & -0.019 & 342.0 & -0.6 & 4 & WSI \\
2003.110 & 0.790 & -0.038 & 342.6 & 0.1 & 2 & ALZ \\
2003.120 & 0.970 & 0.142 & 342.1 & -0.4 & 2 & CTT \\
2007.189 & 0.817 & 0.003 & 339.9 & -0.2 & 1 & SCA \\
2007.195 & 0.813 & -0.001 & 340.7 & 0.6 & 1 & SCA \\
\hline
\end{tabular}

twice with PISCO at OAB in 2007. Since its discovery, the companion has described an arc of about $60^{\circ}$, sufficient for the computation of the preliminary orbital elements reported in Table 1 . As shown in Fig. 2d, the observations seem to indicate a long period. The corresponding systemic mass with the Hipparcos parallax is $4.3 \mathfrak{M}_{\odot}$, in good agreement with the expected value for a system of spectral type A2-4 IV, i.e., $3.7 \mathfrak{M}_{\odot}$.

\section{WDS 10205 +0626 - STF 1426 AB - ADS 7730}

This couple was discovered by F.G.W. Struve in 1832 while he was observing the system H II 43, formerly catalogued as a binary by W. Herschel, and he noted "...Sed major ipsa ex duabus composita in mensuris apparuit..."1(Struve, 1837). The available observations are numerous and of good quality. It was observed with PISCO at OAB in 2004 and 2006. Duflot et al.(1995) report a spectral type $\mathrm{gF} 7$ and a radial velocity of $+2.3 \mathrm{~km} . \mathrm{s}^{-1}$.

Our orbit must be considered as preliminary (grade 4) because the observations cover an arc of only $55^{\circ}$ (Fig. 2e). The parallax measured by Hipparcos with a very large uncertainty (about $85 \%$ !) leads to a systemic mass of $3.4 \mathfrak{M}_{\odot}$, which is consistent with that expected for a spectral type F5-7 IV (2.6 $\left.\mathfrak{M}_{\odot}\right)$.

\section{WDS $19019+1910$ - STF 2437 - ADS 11956}

This binary was measured for the first time by F.G.W. Struve, in 1828, using the famous 9.6-inch refractor of the Dorpat Observatory. The observations

1 "...But the primary star itself appeared as composite during the measurements..." (Struve was referring to the binary system H II 43)
Table $8 \quad O-C$ residuals for the new orbit of STF 1426 AB - ADS 7730

\begin{tabular}{|c|c|c|c|c|c|c|}
\hline Epoch & $\begin{array}{c}\rho \\
\left({ }^{\prime \prime}\right)\end{array}$ & $\begin{array}{c}\Delta \rho_{(O-C)} \\
\left({ }^{\prime \prime}\right)\end{array}$ & $\begin{array}{c}\theta \\
\left(^{\circ}\right)\end{array}$ & $\begin{array}{c}\Delta \theta_{(O-C)} \\
\left({ }^{\circ}\right)\end{array}$ & $\mathrm{n}$ & Observer \\
\hline 1991.250 & 0.960 & 0.033 & 306.0 & -0.1 & 1 & HIP \\
\hline 1991.251 & 0.960 & 0.033 & 304.2 & -1.9 & 1 & WSI \\
\hline 1991.254 & 0.920 & -0.007 & 304.6 & -1.5 & 1 & WSI \\
\hline 1991.262 & 0.940 & 0.013 & 305.8 & -0.3 & 1 & WSI \\
\hline 1991.700 & 0.930 & 0.004 & 308.9 & 2.7 & 1 & TYC \\
\hline 1992.311 & 1.020 & 0.094 & 304.8 & -1.6 & 1 & WSI \\
\hline 1993.327 & 0.780 & -0.145 & 307.7 & 1.0 & 1 & WSI \\
\hline 1993.346 & 0.950 & 0.025 & 306.1 & -0.6 & 1 & WSI \\
\hline 1995.210 & 0.910 & -0.013 & 307.1 & -0.1 & 1 & WSI \\
\hline 1995.317 & 0.910 & -0.013 & 307.5 & 0.3 & 1 & WSI \\
\hline 1997.234 & 0.840 & -0.081 & 309.3 & 1.6 & 1 & WSI \\
\hline 2000.310 & 0.940 & 0.022 & 308.8 & 0.3 & 2 & ALZ \\
\hline 2002.320 & 0.960 & 0.045 & 311.6 & 2.4 & 2 & CTT \\
\hline 2004.304 & 0.932 & 0.019 & 309.2 & -0.5 & 1 & $\mathrm{SCA}$ \\
\hline 2006.283 & 0.929 & 0.019 & 309.0 & -1.3 & 1 & SCA \\
\hline 2006.308 & 0.925 & 0.015 & 309.4 & -0.9 & 1 & SCA \\
\hline
\end{tabular}

Table $9 \quad O-C$ residuals for the new orbit of STF 2437 - ADS 11956

\begin{tabular}{ccrrrll}
\hline Epoch & $\begin{array}{c}\rho \\
\left({ }^{\prime \prime}\right)\end{array}$ & $\begin{array}{c}\Delta \rho_{(O-C)}\left({ }^{\prime \prime}\right) \\
\left({ }^{\circ}\right)\end{array}$ & $\begin{array}{r}\Delta \theta_{(O-C)}\left({ }^{\circ}\right) \\
\text { n }\end{array}$ & Observer \\
\hline 1991.250 & 0.612 & 0.010 & 19.9 & -0.7 & 1 & HIP \\
1991.660 & 0.600 & -0.001 & 23.2 & 2.9 & 1 & TYC \\
1991.667 & 0.590 & -0.011 & 18.5 & -1.8 & 1 & WSI \\
1991.670 & 0.600 & -0.001 & 19.8 & -0.5 & 1 & WSI \\
1991.741 & 0.590 & -0.011 & 19.1 & -1.2 & 1 & WSI \\
1994.630 & 0.520 & -0.075 & 20.2 & 1.6 & 2 & ALZ \\
1994.717 & 0.580 & -0.015 & 14.5 & -4.0 & 1 & WSI \\
1996.650 & 0.660 & 0.068 & 18.7 & 1.4 & 2 & HEI \\
1997.610 & 0.480 & -0.110 & 14.9 & -1.8 & 2 & ALZ \\
2000.620 & 0.590 & 0.005 & 15.0 & 0.1 & 2 & ALZ \\
2003.640 & 0.550 & -0.030 & 15.7 & 2.8 & 2 & ALZ \\
2005.662 & 0.592 & 0.015 & 11.4 & -0.2 & 1 & SCA \\
2006.743 & 0.579 & 0.004 & 11.2 & 0.3 & 1 & SCA \\
\hline
\end{tabular}

are abundant although a little scattered. It was observed with PISCO at OAB in 2005 and 2006. Stephenson and Sanwal (1969), following the complex method proposed by Russell and Moore (1940), and with the data available at that time (magnitude, spectral type, trigonometrical parallax etc.), computed for this system a mass of $1.5 \mathfrak{M}_{\odot}$. Since discovery, the companion has covered an arc of $70^{\circ}$ (see Fig. 2f), sufficient for the computation of the preliminary orbital elements presented in Table 1 . The systemic mass of $0.6 \mathfrak{M}_{\odot}$ derived with the parallax measured by Hipparcos is in default for a system of spectral type G5 IV, for which $2.3 \mathfrak{M}_{\odot}$ is expected (see Table 3). Conversely, the smaller dynamical parallax of 7.8 mas would lead to a systemic mass of $2.5 \mathfrak{M}_{\odot}$, in very good agreement with the theoretical value. 


\section{Comparison with Novakovic (2006)'s orbits}

Shortly after computing those orbits, we learnt that new orbits for ADS 1345, 4376 and 7730 had been independently proposed by Novakovic (2006), which happened to be very different from ours, as shown in Fig. 2. The representation of the observations is almost equivalent for the two sets of orbits with a similar level of the $O-C$ residuals. For ADS 1345 and 7730, the systemic masses derived from Novakovic's orbits are $4.5 \mathfrak{M}_{\odot}$ and $3.5 \mathfrak{M}_{\odot}$, respectively, which is very similar to our estimation. For ADS 4376, Novakovic's estimate is $\mathfrak{M}_{\text {syst }}=5.2 \mathfrak{M}_{\odot}$, which is in excess with the expected mass for A2-4 IV stars $\left(\approx 3.7 \mathfrak{M}_{\odot}\right)$, whereas our estimate (i.e., $4.3 \mathfrak{M}_{\odot}$ ) is in better agreement. Further we note that the residuals of ADS 4376 in $\theta$ are systematically positive after 1976 for Novakovic's orbit, which is not the case for our orbit (see Table 7).

Future observations, covering a greater arc of orbit, will be required to discriminate between the two sets of orbits. For ADS 1345, this should be possible rather quickly (see Fig. 2a), in the next decade or so, but a longer monitoring will be needed for ADS 4376 and 7730 , since the divergence of the ephemerides will not occur before approximately 2050 in both cases.

Finally note that for the three orbits we have in common with Novakovic, the PISCO observations give, on average, a two year advantage in the range of epochs available - i.e. Novakovic's latest observations are $2003 / 4$ whilst ours are 2006/7.

\section{Conclusion}

The six orbits presented here are to be considered as preliminary either because of the restricted arc covered by the observations or, in the case of ADS 3610, because of the small number of observations and their dispersion (see Fig. 2b). With the exception of the orbit of A 1 - ADS 1345 which is the revision of an already published orbit, the orbits of the five other systems, namely A 2629 - ADS 3610, BU 560 - ADS 4371, STF 3115 - ADS 4376, STF 1426 AB - ADS 7730 and STF 2437 - ADS 11956, are new orbits. In the cases of ADS 1345, 4376 and 7730, other orbits for which we have had only an a posteriori knowledge, were also computed independently by Novakovic (2006) and roughly at the same time, but without the PISCO measurements made in Merate. Although the representation of the observations is nearly equivalent between the orbits of Novakovic and ourselves, the orbital elements are quite different. Future observations, covering an extended arc of orbit, should allow an easy discrimination between them.

Five of the orbits published here have been computed with the contribution of observations made at
INAF-OAB with the speckle camera PISCO. Since the installation of PISCO on the 102-cm Zeiss telescope in 2004, this raises to fourteen the total number of the published orbits, that we have computed with measurements obtained in Merate (Scardia et al. 2005, 2006, 2007a,b, 2008).

Acknowledgements. We thank the members of the United States Naval Observatory, Washington DC, for kindly sending on request some lists of measurements of visual binaries. This work has made use of the "Fourth Catalogue of Interferometric Measurements of Binary Stars" (http://ad.usno. navy.mil/wds/int4), the "Sixth Catalogue of Orbits of Visual Binary Stars" (http://ad.usno.navy.mil/wds/orb6), the Washington Double Star Catalogue maintained at the U.S. Naval Observatory (http://ad.usno.navy.mil/wds/wds). and the "Centre de Données Stellaires" (http://simbad.u-strasbg.fr) of Strasbourg (France).

\section{References}

Angelov, T.: 1993, Bull. Astron. Belgrade n. 148, 1

Baize, P.: 1947, Bull. Astron. de Paris, 13, 123

Baize, P., Romani, L.: 1945, Ann. Astrophys., 9, 13

Couteau, P.: 1978, L'observation des étoiles doubles visuelles, Flammarion Ed.

Duflot, M., Figon, P., Meyssonier, N.: 1995, A\&AS 114,269

Erceg, V.: 1981, IAU Comm. 26, Inf. Circ. n. 85

ESA, 1997, The Hipparcos and Tycho Catalogues, ESA SP1200, ESA Publications Division, Noordwijk

Hellerich J.: 1925, Astron. Nachr. 223, 335

Kowalsky M.: 1873, Procès-verbeaux de l'Université Imperiale de Kasan

Mason B.D., Wycoff G.L., Hartkopf W.I.: 2007, "Washington Double Star Catalogue" http://ad.usno.navy.mil/wds/wds.html

Novakovic, B.: 2006, IAU Comm. 26, Inf. Circ. n. 158

Prieur J.-L., Koechlin L., André C., Gallou G., Lucuix C.: 1998, Experimental Astronomy, Vol 8, Issue 4, 297

Royer, F., Grenier, S., Baylac, M.-O., Gomez, A.E., Zorec, J.: 2002, A\&A 393, 897

Russell, H.N., Moore, C.E.: 1940, The masses of the stars, University of Chicago Press, Chicago

Scardia, M.: 1991, A\&AS 90, 565

Scardia, M., Prieur, J.-L., Aristidi, E., Koechlin, L.: 2000, Astron. Nachr. 321, 255

Scardia, M., Prieur, J.-L., Sala, M., Ghigo, M., Koechlin, L., Aristidi, E., Mazzoleni, F.: 2005, MNRAS 357, 1255

Scardia M., Prieur J.-L., Pansecchi L., Argyle R. W., Sala M., Ghigo M., Koechlin L., Aristidi E.: 2006, MNRAS, 367,1170

Scardia M., Prieur J.-L., Pansecchi L., Argyle R. W., Basso S., Sala M., Ghigo M., Koechlin L., Aristidi E.: 2007a, MNRAS, 374, 965

Scardia M., Argyle R. W., Prieur J.-L., Pansecchi L., Basso S., Law N. M., Mackay C.D.: 2007b, Astron. Nachr., $328,2,146$

Scardia M., Prieur J.-L., Pansecchi L., Argyle R., Sala M., Basso S., Ghigo M., Koechlin L., Aristidi E.: 2008, Astron. Nachr., 329, 1, 54

Schmidt-Kaler Th., 1982, in Landolt-Börnstein, Numerical Data and Functional Relationships in Science and Tech- 
nology, K. Schaifers \& H.H. Voigt eds., New Series, Gr. VI, Vol. 2-b (Springer-Verlag, Berlin), p 1

Stephenson, C.B., Sanwal, N.B.: 1969, AJ 74, 689

Straizys, V., Kuriliene, G.: 1981, Astrophys. Space Sci. 80, 353

Struve, F.G.W.: 1827, Catalogus Novus Stellarum duplicium et multiplicium, Schuenmann Ed.

Struve, F.G.W.: 1837, Stellarum duplicium et multiplicium mensurae micrometricae, Typographia Academica Petropoli 